\title{
Mononeuropatía femoral de etiología poco habitual
}

\author{
Coca Benito D. ${ }^{1}$, Torres León JM. ${ }^{2}$, Artigas Montañes O. ${ }^{3}$, Hijosa Pedregosa M. ${ }^{4}$
}

Sanid. mil. 2015; 71 (3): 203-204; ISSN: 1887-8571

Varón de 88 años que acude al servicio de Urgencias por un dolor localizado a nivel de la región inguinal izquierda. Refería dificultad progresiva para la marcha por pérdida de fuerza en la pierna izquierda y decía que tenía "acorchado" la parte interna de ese muslo. El paciente recibía tratamiento anticoagulante con acenocumarol por un antecedente de fibrilación auricular paroxística. Hacía dos semanas había sufrido un traumatismo en el abdomen al golpearse con la barandilla de una escalera. Además, refería astenia y disnea al realizar esfuerzos.

Se encontraba estable hemodinámicamente, el murmullo vesicular estaba conservado, su corazón latía rítmico a 90 1.p.m. El flanco abdominal izquierdo aparecía algo empastado y doloroso a la palpación. El miembro inferior izquierdo se encontraba semiflexionado en posición antiálgica, el signo del psoas era positivo, no era capaz de flexionar la cadera contra resistencia, la sensibilidad táctil y dolorosa estaban disminuidas en la cara anterior e interna del muslo, y el reflejo rotuliano estaba preservado.

Se realizó un hemograma y una bioquímica sérica en la que destacaban la presencia de una anemia normocítica (hemoglobina $10 \mathrm{~g} / \mathrm{dl}$ y hematocrito $30.1 \%$ ) unas cifras elevadas de CPK $(578 \mathrm{u} / \mathrm{l})$ y una función renal alterada (urea $122 \mathrm{mg} / \mathrm{dl}$, creatinina $1.64 \mathrm{mg} / \mathrm{dl})$. El estudio de coagulación revelaba un alargamiento del tiempo de protrombina (INR 5,87) que se atribuyó a la sobredosificación con acenocumarol.

Se realizó un electromiograma de miembros inferiores con objeto de aclarar la semiología descrita, que fue informado como presencia de una lesión neuroapráxica proximal del nervio femoral de carácter moderado.

La imagen aportada por una TC abdominal nos aclaró la etiología del cuadro.

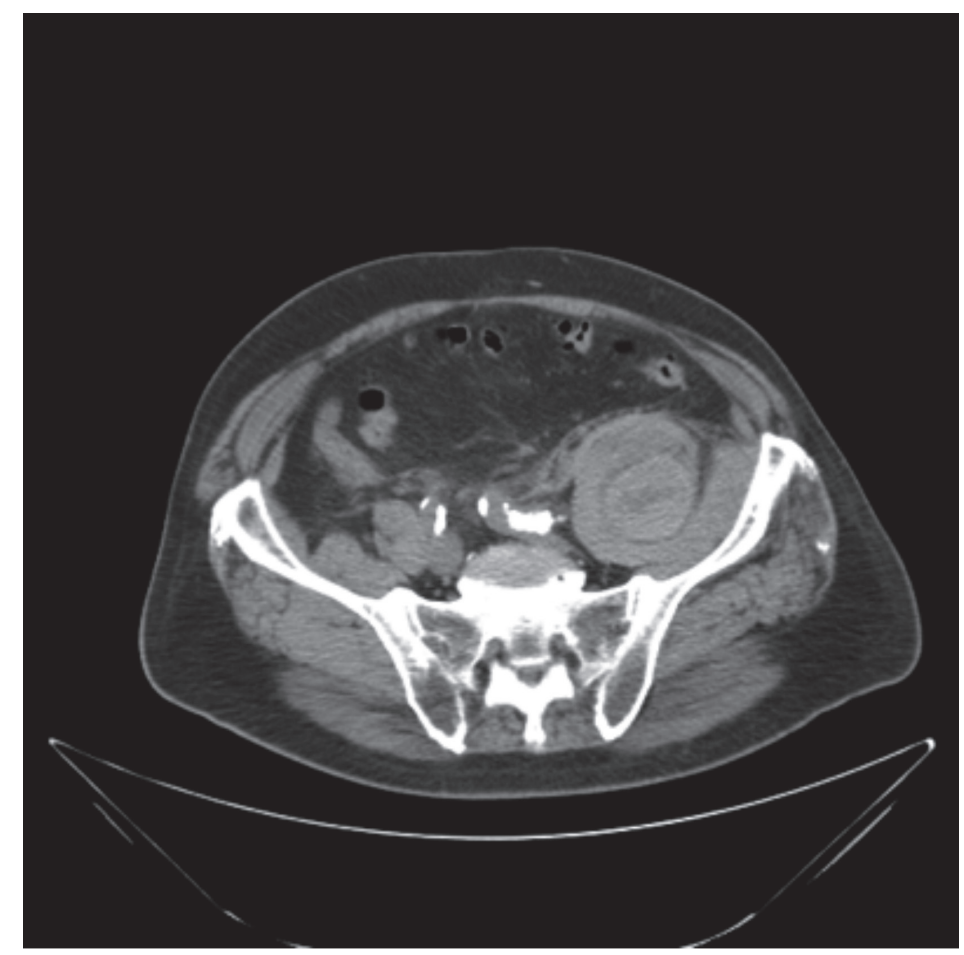

${ }^{1}$ Cte. Médico. Servicio de Medicina Interna.

${ }^{2}$ Tcol. Médico. Servicio de Medicina Interna.

${ }^{3}$ Médico civil. Servicio de Urgencias.

${ }^{4}$ Tcol. Médico. Servicio de Neurofisiología.

Hospital Central de la Defensa Gómez Ulla. Madrid. España.

Dirección para correspondencia: dcocben@oc.mde.es

Recibido: 28 de noviembre de 2014

Aceptado: 8 de junio de 2015 


\section{Diagnóstico: Hematoma retroperitoneal localizado en el músculo psoas-ilíaco}

En los pacientes con fibrilación auricular y riesgo de ictus los beneficios de la anticoagulación oral son indudables, sin embargo la aparición de complicaciones hemorrágicas no son infrecuentes y pueden llegar a ser muy graves. En estos enfermos, se consideran complicaciones hemorrágicas mayores aquellas que causan la muerte, las que requiere hospitalización o la administración de dos o más concentrados de hematíes y las que se producen en determinadas localizaciones como las intracraneales, intraoculares, intraespinales o retroperitoenales ${ }^{1,2}$.

El retroperitoneo es un espacio muy vascularizado que puede albergar grandes hematomas tras complicaciones derivadas de procedimientos quirúrgicos o vasculares, traumatismos, o producirse de forma espontánea en pacientes con discrasias sanguíneas o que reciben tratamiento anticoagulante ${ }^{3}$.

Los hematomas retroperitonales pueden localizarse en los músculos psoas e iliaco. Los síntomas y signos que traducen su existencia suelen ser poco específicos, pueden ser sospechados por la aparición de dolor en el flanco abdominal irradiado a la región lumbar o inguinal, la presencia de una masa palpable o la aparición de inestabilidad hemodinámica inexplicada, sobre todo en pacientes con un factor de riesgo como la anticoagulación. En algunas ocasiones, su presentación resulta aún más atípica y la manifestación dominante es consecuencia del atrapamiento del nervio femoral dentro del hematoma. Un nervio originado en las ramas posteriores de los nervios lumbares $2^{\circ}, 3^{\circ}$ y $4^{\circ}$ y que desciende a través de las fibras del musculo psoas mayor hasta pasar por debajo del ligamento inguinal. Su compresión produce debilidad para la flexión de la cadera y la extensión del muslo, hiopestesia en las zonas anterior del muslo y medial de la pierna y disminución o abolición del reflejo rotuliano, dato que no se apreció en nuestro enfermo probablemente por la lesión incompleta del nervio.

En el caso que se presenta, el paciente refería el antecedente de un traumatismo considerado de poca importancia, al que se añadía un control de anticoagulación muy por encima del rango terapéutico. Los síntomas que motivaron su consulta en el servicio de Urgencias fueron los derivados de la afectación neurológica, aunque en el curso de su asistencia otros datos exploratorios y complementarios, ayudaron a establecer la sospecha diagnóstica confirmada por la imagen presentada. La TC mostraba un gran hematoma retroperitoneal, dependiente del musculo psoas ilíaco, desde L1 hasta pelvis mayor, cuya densidad radiológica era heterogénea por las diferentes fases en la evolución cronológica del hematoma.

El tratamiento de los hematomas del iliaco asociados a neuropatía femoral resulta controvertido, en parte por la rareza de tal afectación. La rapidez en la presentación de los síntomas, el volumen del hematoma y el grado de afectación neurológica son factores que influyen en la estrategia más adecuada. En casos de grandes hematomas, con lesión progresiva del nervio, puede estar indicado el drenaje quirúrgico ${ }^{4} \mathrm{o}$ la descompresión percutánea del mismo a pesar de la dificultad que ofrece el drenaje percutáneo de los hematomas ${ }^{5}$. En casos de sangrado activo, la embolización arterial puede detener la hemorragia. El tratamiento conservador es una opción adecuada en pacientes estables, con síntomas neurológicos moderados y sin sangrado activo $^{6}$. En el caso de nuestro paciente se revirtió la anticoagulación con vitamina $\mathrm{K}$ intravenosa. No presentó datos de inestabilidad hemodinámica, aunque en el curso de su ingreso se transfundieron dos concentrados de hematíes. La afectación del nervio femoral se resolvió por completo en el plazo de una semana. A su alta la TC de control mostró una reducción considerable del hematoma.

\section{BIBLIOGRAFÍA}

1. Palareti G, Cosmi B. Bleeding with anticoagulation therapy-Who is at risk, and how best to identify such patients. Thromb Haemost 2009;102: 268-78.

2. Hughes M, Lip GY, Guideline Development Group for the NICE national clinical guideline for management of atrial fibrillation in primary and secondary care. Risk factors for anticoagulation-related bleeding complications in patients with atrial fibrillation: a systematic review. QJM 2007; 100:599-607.

3. Kasotakis G. Retroperitoneal and rectus sheath hematomas. Surg Clin North Am. 2014 Feb; 94(1):71-6.

4. Weiss JM, Tolo V. Femoral nerve palsy following iliacus hematoma. Orthopedics 2008;31:178.

5. Merrick HW, Zeiss J, Woldenberg LS. Percutaneous decompression for femoral neuropathy secondary to heparin-induced retroperitoneal hematoma: case report and review of the literature. Am Surg 1991; 57:706-11.

6. Patel A, Calfee R, Thakur N, Eberson C. Non-operative management of femoral neuropathy secondary to a traumatic iliacus haematoma in an adolescent. J Bone Joint Surg Br 2008;90:1380-1. 